\title{
A TB Model with Infectivity in Latent Period and Imperfect Treatment
}

\author{
Juan Wang, Sha-Sha Gao, and Xue-Zhi Li \\ Department of Mathematics, Xinyang Normal University, Xinyang 464000, China \\ Correspondence should be addressed to Xue-Zhi Li, xzli66@126.com
}

Received 12 October 2011; Revised 29 December 2011; Accepted 7 January 2012

Academic Editor: Zhen Jin

Copyright (C 2012 Juan Wang et al. This is an open access article distributed under the Creative Commons Attribution License, which permits unrestricted use, distribution, and reproduction in any medium, provided the original work is properly cited.

An epidemiological model of TB with infectivity in latent period and imperfect treatment is introduced. As presented, sustained oscillations are not possible and the endemic proportions either approach the disease-free equilibrium or an endemic equilibrium. The expanded model that stratified the infectious individuals according to their time-since-infection $\theta$ is also carried out. The global asymptotic stability of the infection-free state is established as well as local asymptotic stability of the endemic equilibrium. At the end, numerical simulations are presented to illustrate the results.

\section{Introduction}

Tuberculosis, or TB, is an infectious bacterial disease caused by Mycobacterium tuberculosis ( $M$. tuberculosis), which most commonly affects the lungs. It is transmitted from person to person via droplets from the throat and lungs of people with the active respiratory disease. Tubercle bacilli carried by such droplets live in the air for a short period of time (about 2 hours), and therefore it is believed that occasional contact with an infectious case rarely leads to an infection [1]. In most cases, the body is able to fight the bacteria to stop them from growing. The bacteria become inactive, but since they remain alive, can become active later. People who are infected with TB do not feel sick, do not have any symptoms, and cannot spread TB. But they could develop TB disease at some time in the future. The symptoms of active TB of the lung are coughing, sometimes with sputum or blood, chest pains, weakness, weight loss, fever, and night sweats.

It is estimated that one-third of the world's population has been infected with the $M$. tuberculosis, which is a major cause of illness and death worldwide, especially in Asia and Africa [2]. 1 in 10 people infected with TB bacilli will become sick with active TB in their lifetime. If not treated, each person with active TB infects on average 10 to 15 people every year. There were 9.4 million new TB cases in 2008 (3.6 million of whom are women) including 
1.4 million cases among people living with HIV, and 1.8 million people died from TB in 2008, including 500000 people with HIV - equal to 4500 deaths a day (WHO, 2009). World TB Day, falling on March 24th each year, is designed to build public awareness that tuberculosis today remains an epidemic in much of the world, causing the deaths of several million people each year, mostly in the third world.

TB is curable and considerable progress has been made in controlling TB in the whole world. 36 million people were cured in DOTS programmes (between 1995-2008), with as many as 8 million deaths averted through DOTS. The $87 \%$ global treatment success rate exceeded the $85 \%$ target for the first time since the target was set in 1991 . However, TB bacteria can become resistant to the medicines used to treat TB disease. This means that the medicine can no longer kill the bacteria. Multidrug-resistant TB (MDR-TB) is a form of TB that is difficult and expensive to treat and fails to respond to standard first-line drugs. Extensively drug-resistant TB (XDR-TB) occurs when resistance to second-line drugs develops on top of MDR-TB. $5 \%$ of all TB cases have MDR-TB, based on data from more than 100 countries collected during the last decade (WHO, 2009).

The transmission dynamics of TB has received considerable attention for a long time, and different mathematical models have been developed incorporating various factors, such as fast and slow progression [1], treatment [3-5], drug-resistant strains [6-8], reinfection [8, 9], coinfection with HIV [10-13], migration [14, 15], chemoprophylaxis [5], relapse [16], exogenous reinfection [17], seasonality [15, 18, 19], and age dependent risks [9].

However, most models mentioned above assume that individuals who are latently infected are neither clinically ill nor capable transmitting TB. In this paper, we assume that people in latent period also have infectivity [20], which may occur with the development of the disease. We can find some ODEs models considering infectivity in latent period [2123], in which [21, 22] are SEI epidemic models, and [23] is SEIR epidemic model. An agestructured MSEIS epidemic model with infectivity in latent period has also been discussed in [20], which stratified each class according to its real age and assume that people in latent and infected period has the same infectiveness. Our work differs from these studies in that we considers weaker infectivity in latent period as well as imperfect treatment. Further, we introduce an age-structured epidemic model in which the infective stage is stratified by the age-since-infection.

The structure of the paper is organized as follows. In Section 2, we formulate a simple ODEs model and prove the globally asymptotical stability of the disease-free equilibrium and the endemic equilibrium, respectively. The basic replacement ratio is also briefly discussed in this section. An extension of the ODEs model from the previous section, that is, an agestructured model, is analyzed in Section 3. In two subsections we, respectively, discuss the globally asymptotical stability of the disease-free equilibrium and the locally asymptotical stability of the endemic equilibrium. The numerical simulations and brief discussion are given in Sections 4 and 5, respectively.

\section{A Simple ODEs Model}

In this section, we begin with a simple ODEs (ordinary differential equations) model with infectivity in latent period and imperfect treatment. Since the disease progression is slow the model should also incorporate demographic changes in the population. It is assumed that the total population is growing exponentially [24]. We consider a population whose total population size at time $t$ is denoted by $N(t)$, which is divided into three 
classes: $S(t)$ —susceptible individuals; $E(t)$ 一latent individuals; $I(t)$-infective individuals, who may receive imperfect treatment and enter $E(t)$ again. In fact, the latent may also receive treatment and recover, thus move to $S(t)$ instead of $I(t)$. In addition, we assume that those who are exposed have infectiousness too, but weaker than that of the infectious ones.

The model takes the form:

$$
\begin{gathered}
S^{\prime}=b N-\Lambda S-\mu S+\alpha E+\gamma_{1} I, \\
E^{\prime}=\Lambda S-(\mu+\delta+\alpha) E+\gamma_{2} I, \\
I^{\prime}=\delta E-\left(\mu+\gamma_{1}+\gamma_{2}\right) I, \\
\Lambda=\frac{\beta(k E+I)}{N},
\end{gathered}
$$

where we have used the following parameters: $b$ : birth/recruitment rate into the population, $\mu$ : per capita natural death rate, $k$ : the coefficient of reduction of infection, $\delta$ : the rate at which exposed individuals become infective, $\alpha$ : per capita recovery rate from the class $E, \gamma_{1}$ : per capita recovery rate from the class $I, \gamma_{2}$ : per capita imperfect treatment rate.

We assume that all parameters are nonnegative and $\mu>0$.

The demographic equation for the dynamics of the total population size $(N=S+E+$ $I)$ is given by:

$$
N^{\prime}=b N-\mu N
$$

We obtain $N=N_{0} e^{r t}$, where $r=b-\mu$. Hence, $r$ gives the growth rate of the population. If $r>0$, that is, $b>\mu$, the population grows exponentially; if $r<0$, that is, $b<\mu$, the population decreases exponentially. The case $r=0$ implies that the population is stationary. These thresholds are often interpreted in terms of the demographic reproduction number.

\subsection{The Threshold}

In this subsection we derive the threshold, namely, the basic reproduction number, by considering the existence of the endemic equilibrium, and then analyze the meaning of each part.

Since the model (2.1) is homogeneous of degree one, we consider the equations for the normalized quantities. Setting $s=S / N, e=E / N, i=I / N$ leads to the following equivalent nonhomogeneous system:

$$
\begin{gathered}
s^{\prime}=b(1-s)-\lambda s+\alpha e+\gamma_{1} i, \\
e^{\prime}=\lambda s-(b+\delta+\alpha) e+\gamma_{2} i, \\
i^{\prime}=\delta e-\left(b+\gamma_{1}+\gamma_{2}\right) i, \\
\lambda=\beta(k e+i),
\end{gathered}
$$


where $s+e+i=1$. It is evident that the system (2.3) always have a DFE (disease-free equilibrium): $P_{0}=(1,0,0)$. To see the existence of the endemic equilibrium, we define

$$
R_{0}=\frac{k \beta}{b+\delta+\alpha}+\frac{\delta \beta}{(b+\delta+\alpha)\left(b+\gamma_{1}+\gamma_{2}\right)}+\frac{\delta \gamma_{2}}{(b+\delta+\alpha)\left(b+\gamma_{1}+\gamma_{2}\right)}
$$

which denotes the basic reproduction number, that is, the average number of secondary infections produced by an infective individual during the entire infectious period in a purely susceptible population. The first term $k \beta /(b+\delta+\alpha)$ can be interpreted as the contribution to the reproduction number due to secondary infections generated by an infective individual when he or she is in the class of $E$, and the second term $\delta \beta /(b+\delta+\alpha)\left(b+\gamma_{1}+\gamma_{2}\right)$ can be decomposed to $(\delta /(b+\delta+\alpha))\left(\beta /\left(b+\gamma_{1}+\gamma_{2}\right)\right)$, where $\delta /(b+\delta+\alpha)$ denotes one move to $I$ from $E$, and $\beta /\left(b+\gamma_{1}+\gamma_{2}\right)$ represents the secondary infections generated by an infective individual when he or she is in the class of $I$. Finally, the third term can also be decomposed to $(\delta /(b+\delta+\alpha))\left(\gamma_{2} /\left(b+\gamma_{1}+\gamma_{2}\right)\right)$, where $\delta /(b+\delta+\alpha)$ denotes one move to $I$ from $E$, and $\gamma_{2} /\left(b+\gamma_{1}+\gamma_{2}\right)$ should be responsible for those who get imperfect treatment and enter $E$ again. For the sake of convenience, $R_{0}$ can be reduced to

$$
R_{0}=\frac{k \beta}{b+\delta+\alpha}+\frac{\delta\left(\beta+\gamma_{2}\right)}{(b+\delta+\alpha)\left(b+\gamma_{1}+\gamma_{2}\right)}
$$

and in particular, when $R_{0}>1$, there is an unique endemic equilibrium $P^{*}=\left(s^{*}, e^{*}, i^{*}\right)$, where

$$
\begin{gathered}
s^{*}=1-e^{*}-i^{*}, \\
e^{*}=\frac{1}{\delta}\left(b+\gamma_{1}+\gamma_{2}\right) i^{*}, \\
i^{*}=\frac{1}{m}\left(R_{0}-1\right), \\
m=\left[\frac{k \beta}{b+\delta+\alpha}+\frac{\delta \beta}{(b+\delta+\alpha)\left(b+\gamma_{1}+\gamma_{2}\right)}\right] \frac{b+\gamma_{1}+\gamma_{2}+\delta}{\delta}>0 .
\end{gathered}
$$

It can be easily seen that $R_{0}$ is an increasing function of $\beta$ and $k$, decreasing function of $b, \gamma_{1}$, and $\alpha$. However, it has more complicated relations with $\delta$ and $\gamma_{2}$. By calculating the derivation we get:

$$
\begin{gathered}
\frac{d R_{0}}{d \delta}=\frac{(b+\alpha)\left(\beta+\gamma_{2}\right)-\left(b+\gamma_{1}+\gamma_{2}\right) k \beta}{(b+\delta+\alpha)^{2}\left(b+\gamma_{1}+\gamma_{2}\right)}, \\
\frac{d R_{0}}{d \gamma_{2}}=\frac{\delta\left(b+\gamma_{1}-\beta\right)}{(b+\delta+\alpha)\left(b+\gamma_{1}+\gamma_{2}\right)^{2}},
\end{gathered}
$$

which imply that $R_{0}$ is an increasing function of $\delta$ if $(b+\alpha)\left(\beta+\gamma_{2}\right)-\left(b+\gamma_{1}+\gamma_{2}\right) k \beta>0$ and a decreasing function of $\delta$ if $(b+\alpha)\left(\beta+\gamma_{2}\right)-\left(b+\gamma_{1}+\gamma_{2}\right) k \beta<0$ as well as that $R_{0}$ is an increasing function of $\gamma_{2}$ if $b+\gamma_{1}-\beta>0$ and a decreasing function of $\gamma_{2}$ if $b+\gamma_{1}-\beta<0$. 
Since $s+e+i=1$, the system (2.3) can be reduced to the following equivalent system by replacing $s$ by $1-e-i$ :

$$
\begin{gathered}
e^{\prime}=\lambda(1-e-i)-(b+\delta+\alpha) e+\gamma_{2} i, \\
i^{\prime}=\delta e-\left(b+\gamma_{1}+\gamma_{2}\right) i, \\
\lambda=\beta(k e+i) .
\end{gathered}
$$

Thus in the following sections, we only need to investigate the properties of the DFE $Q_{0}=$ $(0,0)$ and endemic states $Q^{*}=\left(e^{*}, i^{*}\right)$ of the system $(2.9)$, where $e^{*}$ and $i^{*}$ have been given in (2.6), which are corresponding to $P_{0}$ and $P^{*}$, respectively.

\subsection{The Globally Asymptotical Stability of the DFE}

In this subsection, we firstly prove that the disease-free equilibrium (DFE) is locally stable when $R_{0}<1$ by calculating the Jacobian of (2.9) at $Q_{0}$, and then choose a proper Liapnov function to get the globally asymptotical stability of the DFE.

Theorem 2.1. If $R_{0}<1$, then $Q_{0}$ is locally asymptotically stable (LAS); if $R_{0}>1$, then $Q_{0}$ is unstable.

Proof. The Jacobian of (2.9) at $Q_{0}$ is

$$
J(0,0)=\left(\begin{array}{cc}
k \beta-(b+\delta+\alpha) & \beta+\gamma_{2} \\
\delta & -\left(b+\gamma_{1}+\gamma_{2}\right)
\end{array}\right) .
$$

It is easy to see that the two eigenvalues $\omega_{1}$ and $\omega_{2}$ satisfy

$$
\begin{gathered}
\omega_{1}+\omega_{2}=k \beta-(b+\delta+\alpha)-\left(b+\gamma_{1}+\gamma_{2}\right), \\
\omega_{1} \omega_{2}=(b+\delta+\alpha)\left(b+\gamma_{1}+\gamma_{2}\right)\left(1-R_{0}\right) .
\end{gathered}
$$

If $R_{0}<1$, then $\omega_{1}+\omega_{2}<0$ and $\omega_{1} \omega_{2}>0$, thus both $\omega_{1}$ and $\omega_{2}$ are negative, which implies $Q_{0}$ is locally asymptotically stable; if $R_{0}>1$, then there is a positive eigenvalue, so $Q_{0}$ is unstable. This completes the proof.

Theorem 2.2. If $R_{0}<1$, the DFE $Q_{0}$ is globally asymptotically stable (GAS).

Proof. We choose a liapunov function

$$
V=\left[\delta+k\left(b+\gamma_{1}+\gamma_{2}\right)\right] e+\left(b+\delta+\alpha+k \gamma_{2}\right) i
$$


It is easy to calculate that

$$
\begin{aligned}
V^{\prime}= & {\left[\delta+k\left(b+\gamma_{1}+\gamma_{2}\right)\right] e^{\prime}+\left(b+\delta+\alpha+k \gamma_{2}\right) i^{\prime} } \\
= & (b+\delta+\alpha)\left(b+\gamma_{1}+\gamma_{2}\right) R_{0}(k e+i)-(e+i)\left[k\left(b+\gamma_{1}+\gamma_{2}\right) \lambda+\delta \lambda\right] \\
& -(b+\delta+\alpha)\left(b+\gamma_{1}+\gamma_{2}\right)(k e+i) \\
= & (b+\delta+\alpha)\left(b+\gamma_{1}+\gamma_{2}\right)(k e+i)\left(R_{0}-1\right) \\
& -\left[k \beta\left(b+\gamma_{1}+\gamma_{2}\right)+\delta \beta\right](e+i)(k e+i) .
\end{aligned}
$$

If $R_{0}<1$, noting that $e \geq 0, i \geq 0$ and all the parameters are positive, then $V^{\prime}<0$. If $V^{\prime}=0$, since

$$
(b+\delta+\alpha)\left(b+\gamma_{1}+\gamma_{2}\right)\left(R_{0}-1\right)-\left[k \beta\left(b+\gamma_{1}+\gamma_{2}\right)+\delta \beta\right](e+i) \neq 0,
$$

it follows from (2.13) that $k e+i=0$, that is, $e=i=0$. So $Q_{0}$ is globally attractive. Coupled with Theorem 2.1, we can derive that $Q_{0}$ is globally asymptotically stable when $R_{0}<1$. The proof is complete

\subsection{The Globally Asymptotical Stability of the Endemic Equilibrium}

In this subsection, we firstly prove that the endemic equilibrium is asymptotically stable if it exists by calculating the Jacobian of (2.9) at $Q^{*}$ and then choose a proper Dulac function to rule out the periodic solution.

Theorem 2.3. If $R_{0}>1$, the endemic equilibrium $Q^{*}$ is locally asymptotically stable (LAS).

Proof. The Jacobian of (2.9) at $Q^{*}$ is

$$
J\left(e^{*}, i^{*}\right)=\left(\begin{array}{cc}
k \beta\left(1-e^{*}-i^{*}\right)-\beta l-(b+\delta+\alpha) & \beta\left(1-e^{*}-i^{*}\right)-\beta l+\gamma_{2} \\
\delta & -\left(b+\gamma_{1}+\gamma_{2}\right)
\end{array}\right),
$$

where $l=k e^{*}+i^{*}$. Since the endemic state obtained from (2.9) by putting the derivatives equal to zero, the $(1,1)$ entry in the Jacobian can be written as

$$
-\frac{\delta}{b+\gamma_{1}+\gamma_{2}}\left[\beta\left(1-e^{*}-i^{*}\right)+\gamma_{2}\right]-\beta l<0
$$

It is easy to see that the two eigenvalues $\omega_{3}$ and $\omega_{4}$ satisfy $\omega_{3}+\omega_{4}<0$ and

$$
\omega_{3} \omega_{4}=\beta l\left(b+\gamma_{1}+\gamma_{2}+\delta\right)>0,
$$

which implies that both the eigenvalues are negative. Thus we can conclude that $Q^{*}$ is locally asymptotically stable. This completes the proof. 
System (2.9) is two dimensional and direct application of the Dulac's criterion is possible. We define the relevant region

$$
\Phi=\{(e, i) \mid e \geq 0, i \geq 0, e+i \leq 1\}
$$

Proposition 2.4. The system (2.9) has no periodic solutions, homoclinic loops, or oriented phase polygons inside the region $\boldsymbol{\Phi}$.

Proof. Let

$$
\begin{gathered}
F(e, i)=\beta(k e+i)(1-e-i)-(b+\delta+\alpha) e+\gamma_{2} i, \\
G(e, i)=\delta e-\left(b+\gamma_{1}+\gamma_{2}\right) i .
\end{gathered}
$$

As a Dulac multiplier we use $1 / e$. We have

$$
\frac{\partial F(e, i) / e}{\partial e}+\frac{\partial G(e, i) / e}{\partial e}=-\frac{\beta i}{e^{2}}(1-e-i)-\beta\left(k+\frac{i}{e}\right)-\frac{\gamma_{2} i}{e^{2}}-\frac{1}{\left(b+\gamma_{1}+\gamma_{2}\right) e}<0,
$$

and therefore there are no closed orbits in the region $\boldsymbol{\Phi}$. This completes the proof.

From Theorem 2.3 and Proposition 2.4, we can immediately obtain the following theorem.

Theorem 2.5. If $R_{0}>1$, the endemic equilibrium $Q^{*}$ is globally asymptotically stable (GAS).

\section{An Age-Structured Model}

In this section we consider an extension of the ODEs model from the previous section in which the infective stage is stratified by the age-since-infection, that is, the time spent in the infective stage. Let $\theta$ be the age-since-infection. With the notation from the previous section we consider

$$
\begin{gathered}
S^{\prime}=b N-\Lambda S-\mu S+\alpha E+\int_{0}^{\infty} \gamma_{1}(\theta) i(\theta, t) d \theta, \\
E^{\prime}=\Lambda S-(\mu+\delta+\alpha) E+\int_{0}^{\infty} \gamma_{2}(\theta) i(\theta, t) d \theta, \\
i_{\theta}+i_{t}=-\left(\mu+\gamma_{1}(\theta)+\gamma_{2}(\theta)\right) i(\theta, t), \\
i(0, t)=\delta E, \\
\Lambda=\frac{\beta\left(k E+\int_{0}^{\infty} i(\theta, t) d \theta\right)}{N}, \\
S(0)=S_{0}, \quad E(0)=E_{0}, \quad i(\theta, 0)=i_{0}(\theta),
\end{gathered}
$$


where $\gamma_{1}(\theta)$ and $\gamma_{2}(\theta)$ are nonnegative functions of $\theta$. As before, $\gamma_{1}(\theta)$ is the age-structured recovery rate for the infective state, and $\gamma_{2}(\theta)$ is the age structured imperfect treatment rate of individuals who have infected. We will use the following notations:

$$
I(t)=\int_{0}^{\infty} i(\theta, t) d \theta, \quad \Gamma_{j}(\theta)=e^{-\int_{0}^{\theta} \gamma_{j}(\tau) d \tau} \quad(j=1,2),
$$

where $I(t)$ is the number of infected individuals, $\Gamma_{1}(\theta)$ represents the probability of not recovering to $S(t)$ at $\theta$ time units after becoming infected, and $\Gamma_{2}(\theta)$ denotes the probability of not receiving imperfect treatment and enter into $E(t)$ again at $\theta$ time units after becoming infected, therefore $\Gamma(\theta)=\Gamma_{1}(\theta) \Gamma_{2}(\theta)$ is the probability of being still infective $\theta$ time units after becoming infected. Integrating the third equation in (3.1) and assuming that there are no individuals with infinite age-since-infection, that is, $i(\theta, t) \rightarrow 0$ when $\theta \rightarrow \infty$ for all $t$, we get

$$
I^{\prime}(t)=\delta E-\mu I-\int_{0}^{\infty}\left(\gamma_{1}(\theta)+\gamma_{2}(\theta)\right) i(\theta, t) d \theta,
$$

thus for the total population size $N=S+E+I$ we obtain a Malthus equation of exponential growth: $N^{\prime}=b N-\mu N$.

Similar to the previous section, by introducing the proportions $s=S / N, u=$ $E / N, v=i / N$ we get the normalized system:

$$
\begin{gathered}
s^{\prime}=b(1-s)-\lambda s+\alpha u+\int_{0}^{\infty} \gamma_{1}(\theta) v(\theta, t) d \theta, \\
u^{\prime}=\lambda s-(b+\delta+\alpha) u+\int_{0}^{\infty} \gamma_{2}(\theta) v(\theta, t) d \theta, \\
v_{\theta}+v_{t}=-\left(b+\gamma_{1}(\theta)+\gamma_{2}(\theta)\right) v(\theta, t), \\
v(0, t)=\delta u, \\
\lambda=\beta\left(k u+\int_{0}^{\infty} v(\theta, t) d \theta\right), \\
s(0)=s_{0}, \quad u(0)=u_{0}, \quad v(\theta, 0)=v_{0}(\theta),
\end{gathered}
$$

where $s(t)+u(t)+\int_{0}^{\infty} v(\theta, t) d \theta=1$.

It is easy to show that (3.4) always exists in a DFE: $M_{0}=(1,0,0)$. Let $M^{*}=$ $\left(s^{*}, u^{*}, v^{*}(\theta)\right)$ be an endemic equilibrium of (3.4), then $M^{*}$ satisfies the following equations:

$$
\begin{aligned}
& 0=b\left(1-s^{*}\right)-\lambda^{*} s^{*}+\alpha u^{*}+\int_{0}^{\infty} \gamma_{1}(\theta) v^{*}(\theta) d \theta, \\
& 0=\lambda^{*} s^{*}-(b+\delta+\alpha) u^{*}+\int_{0}^{\infty} \gamma_{2}(\theta) v^{*}(\theta) d \theta \\
& v_{\theta}^{*}(\theta)=-\left(b+\gamma_{1}(\theta)+\gamma_{2}(\theta)\right) v^{*}(\theta), \\
& v^{*}(0)=\delta u^{*}, \\
& \lambda^{*}=\beta\left(k u^{*}+\int_{0}^{\infty} v^{*}(\theta) d \theta\right)
\end{aligned}
$$

where $s^{*}+u^{*}+\int_{0}^{\infty} v^{*}(\theta) d \theta=1$. 
From the third and fourth equations of above system it follows that

$$
v^{*}(\theta)=\delta u^{*} e^{-b \theta} \Gamma(\theta)
$$

From this and the following equation

$$
\beta\left(k u^{*}+\int_{0}^{+\infty} v^{*}(\theta) d \theta\right) s^{*}-(b+\delta+\alpha) u^{*}+\int_{0}^{+\infty} r_{2}(\theta) v^{*}(\theta)=0
$$

we get $M^{*}=\left(s^{*}, u^{*}, v^{*}(\theta)\right)$ can be represented by

$$
\begin{gathered}
s^{*}=\frac{b+\delta+\alpha-\delta \int_{0}^{\infty} \gamma_{2}(\theta) e^{-b \theta} \Gamma(\theta) d \theta}{\beta\left(k+\delta \int_{0}^{\infty} e^{-b \theta} \Gamma(\theta) d \theta\right)}, \\
u^{*}=\frac{1-s^{*}}{1+\delta \int_{0}^{\infty} e^{-b \theta} \Gamma(\theta) d \theta^{\prime}}, \\
v^{*}(\theta)=\delta u^{*} e^{-b \theta} \Gamma(\theta) .
\end{gathered}
$$

\subsection{The Globally Asymptotical Stability of the DFE}

In this subsection, to analyze the stability of the DFE we still take the linearization of system (3.4) at the point $M_{0}$ as before and obtain the threshold $R_{0}$, namely, the basic reproduction number, then we prove that the DFE is globally attractive if $R_{0}<1$.

Theorem 3.1. If $R_{0}<1$, the disease-free equilibrium $M_{0}$ is locally asymptotically stable (LAS); if $R_{0}>1, M_{0}$ is unstable and there is a unique endemic equilibrium $M^{*}$.

Proof. Set

$$
s=1+\bar{x}, \quad u=\bar{y}, \quad v=\bar{z}
$$

Plug it into the system (3.4) and ignore the high-degree terms, then we get the linearization around DFE $M_{0}$ :

$$
\begin{gathered}
\bar{x}^{\prime}=-b \bar{x}-\bar{\lambda}+\alpha \bar{y}+\int_{0}^{\infty} \gamma_{1}(\theta) \bar{z}(\theta, t) d \theta \\
\bar{y}^{\prime}=\bar{\lambda}-(b+\delta+\alpha) \bar{y}+\int_{0}^{\infty} \gamma_{2}(\theta) \bar{z}(\theta, t) d \theta \\
\bar{z}_{\theta}+\bar{z}_{t}=-\left(b+\gamma_{1}(\theta)+\gamma_{2}(\theta)\right) \bar{z}(\theta, t) \\
\bar{z}(0, t)=\delta \bar{y} \\
\bar{\lambda}=\beta\left(k \bar{y}+\int_{0}^{\infty} \bar{z}(\theta, t) d \theta\right) .
\end{gathered}
$$


Looking for exponential solutions in (3.10), that is, solutions of the form

$$
\bar{x}=e^{\omega t} x, \quad \bar{y}=e^{\omega t} y, \quad \bar{z}=e^{\omega t} z(\theta),
$$

where $\omega$ is a constant. Substituting it into (3.10), we have

$$
\begin{gathered}
\omega x=-b x-\tilde{\lambda}+\alpha y+\int_{0}^{\infty} \gamma_{1}(\theta) z(\theta) d \theta, \\
\omega y=\tilde{\lambda}-(b+\delta+\alpha) y+\int_{0}^{\infty} \gamma_{2}(\theta) z(\theta) d \theta, \\
\omega z+z_{\theta}=-\left(b+\gamma_{1}(\theta)+\gamma_{2}(\theta)\right) z(\theta), \\
z(0)=\delta y, \\
\tilde{\lambda}=\beta\left(k y+\int_{0}^{\infty} z(\theta) d \theta\right) .
\end{gathered}
$$

From the third and the forth equations in (3.12), we get $z(\theta)=\delta y e^{-(\omega+b) \theta} \Gamma(\theta)$. The characteristic equation is:

$$
\omega=k \beta+\beta \delta \int_{0}^{\infty} e^{-(\omega+b) \theta} \Gamma(\theta) d \theta-(b+\delta+\alpha)+\delta \int_{0}^{\infty} \gamma_{2}(\theta) e^{-(\omega+b) \theta} \Gamma(\theta) d \theta
$$

which can be rewritten in the form

$$
\frac{k \beta+\delta \int_{0}^{\infty}\left(\beta+\gamma_{2}(\theta)\right) e^{-(\omega+b) \theta} \Gamma(\theta) d \theta}{\omega+b+\delta+\alpha}=1
$$

Denote the left hand side of (3.14) by $F(\omega)$ and define the basic reproduction number

$$
R_{0}=F(0)=\frac{k \beta+\delta \int_{0}^{\infty}\left(\beta+\gamma_{2}(\theta)\right) e^{-b \theta} \Gamma(\theta) d \theta}{b+\delta+\alpha}
$$

For $\omega \leq-(b+\delta+\alpha), F(\omega)$ is negative and the equation has no solution. For $\omega>-(b+\delta+\alpha)$, $F(\omega)$ is a decreasing function for real $\omega$ which approaches $\infty$ as $\omega \rightarrow-(b+\delta+\alpha)$ and zero as $\omega \rightarrow \infty$. Therefore, the characteristic equation always has a unique real solution $\omega^{*}$, and if 
$R_{0}<1$, then $\omega^{*}<0$; if $R_{0}>1$, then $\omega^{*}>0$, which implies that the DFE is unstable. In addition we assume that $\omega=c+d i$ is an arbitrary complex solution of (3.14), then we have

$$
\begin{aligned}
1 & =F(\omega)=F(c+d i)=|F(c+d i)| \\
& =\left|\frac{k \beta+\delta \int_{0}^{\infty}\left(\beta+\gamma_{2}(\theta)\right) e^{-(\omega+b) \theta} \Gamma(\theta) d \theta}{\omega+b+\delta+\alpha}\right| \\
& =\frac{k \beta+\delta \int_{0}^{+\infty}\left(\beta+\gamma_{2}(\theta) e^{-(b+c) \theta} \Gamma(\theta) \cos (d \theta)\right) d \theta}{c+b+\delta+\alpha} \\
& \leq \frac{k \beta+\delta \int_{0}^{+\infty}\left(\beta+\gamma_{2}(\theta) e^{-(b+c) \theta} \Gamma(\theta)\right) d \theta}{c+b+\delta+\alpha} \\
& =F(c) .
\end{aligned}
$$

Since $F(x)$ is a decreasing function for real $x$ and $\omega^{*}$ satisfies (3.14), we have that $c \leq \omega^{*}$. Hence, any complex solution of (3.14) has a real part smaller than the unique real solution of (3.14). Therefore, if $R_{0}<1$, then the disease-free equilibrium is locally asymptotically stable.

We note that if $R_{0}<1$, then $s^{*}>1$ (see (3.8)), thus the endemic state does not exist. If $R_{0}>1$, then $s^{*}$ as given by (3.8) is smaller than one and an endemic state exists and is given by (3.8). This completes the proof.

If we can show that the DFE is globally attractive, then coupled with the above theorem we derive the DFE is globally asymptotically stable (GAS). In particular, we have the following theorem.

Theorem 3.2. Assume that $\gamma_{2}(\theta)$ is a bounded function and

$$
\bar{\gamma}_{2}=\sup _{\theta \in[0, \infty)} \gamma_{2}(\theta)
$$

If $R_{0}<1$, the disease-free equilibrium $M_{0}$ is globally asymptotically stable (GAS).

Proof. Integrating the third equation of (3.4) along the characteristic lines we obtain

$$
v(\theta, t)= \begin{cases}v_{0}(\theta-t) e^{-b t} \Gamma(\theta-t, \theta), & \theta \geq t \\ \delta u(t-\theta) e^{-b \theta} \Gamma(\theta), & \theta<t\end{cases}
$$

where $\Gamma(\theta-t, \theta)=e^{-\int_{\theta-t}^{\theta}\left(\gamma_{1}(\tau)+\gamma_{2}(\tau)\right) d \tau}$ is the probability of an individual who has been infected (in class $I)$ ) for $\theta-t$ units to remain infective until $\theta$ units after infection. Then

$$
\begin{aligned}
\int_{0}^{\infty} \gamma_{2}(\theta) v(\theta, t) d \theta & =\int_{0}^{t} \gamma_{2}(\theta) \delta u(t-\theta) e^{-b \theta} \Gamma(\theta) d \theta+\int_{t}^{\infty} \gamma_{2}(\theta) v_{0}(\theta-t) e^{-b t} \Gamma(\theta-t, \theta) d \theta \\
& \leq \int_{0}^{t} \gamma_{2}(\theta) \delta u(t-\theta) e^{-b \theta} \Gamma(\theta) d \theta+\bar{\gamma}_{2}\left\|v_{0}\right\|_{L^{1}} e^{-b t}
\end{aligned}
$$


Taking the upper limit for $t \rightarrow \infty$ in the above inequality leads to

$$
\limsup _{t \rightarrow \infty} \int_{0}^{\infty} \gamma_{2}(\theta) v(\theta, t) d \theta \leq \int_{0}^{\infty} r_{2}(\theta) \delta e^{-b \theta} \Gamma(\theta) d \theta \limsup _{t \rightarrow \infty} u(t) .
$$

From the second equation in (3.4) and using the fact that $s \leq 1$ we obtain

$$
\begin{aligned}
u(t) & =u_{0} e^{-(b+\delta+\alpha) t}+\int_{0}^{t}\left[\beta k s u(\tau)+\int_{0}^{\infty}\left(\beta s+\gamma_{2}(\theta)\right) v(\theta, \tau) d \theta\right] e^{-(b+\delta+\alpha)(t-\tau)} d \tau \\
& \leq u_{0} e^{-(b+\delta+\alpha) t}+\int_{0}^{t}\left[\beta k u(t-\tau)+\int_{0}^{\infty}\left(\beta+\gamma_{2}(\theta)\right) v(\theta, t-\tau) d \theta\right] e^{-(b+\delta+\alpha) \tau} d \tau .
\end{aligned}
$$

Taking the upper limit for $t \rightarrow \infty$ in the above inequality we get

$$
\begin{aligned}
\limsup _{t \rightarrow \infty} u(t) \leq & \limsup _{t \rightarrow \infty} u_{0} e^{-(b+\delta+\alpha) t} \\
& +\limsup _{t \rightarrow \infty} \int_{0}^{t}\left[\beta k u(t-\tau)+\int_{0}^{+\infty}\left(\beta+\gamma_{2}(\theta)\right) v(\theta, t-\tau) d \theta\right] e^{-(b+\delta+\alpha) \tau} d \tau \\
\leq & \int_{0}^{+\infty}\left[\beta k \limsup _{t \rightarrow \infty} u(t-\tau)+\limsup _{t \rightarrow \infty} \int_{0}^{t}\left(\beta+\gamma_{2}(\theta)\right) \delta u(t-\theta-\tau) e^{-b \theta} \Gamma(\theta) d \theta\right. \\
& \left.+\limsup _{t \rightarrow \infty} \int_{t}^{+\infty}\left(\beta+\gamma_{2}(\theta)\right) v_{0}(\theta-t+\tau) e^{-b(t-\tau)} \Gamma(\theta-t+\tau, \theta) d \theta\right] e^{-(b+\delta+\alpha) \tau} d \tau \\
= & \int_{0}^{+\infty}\left[\beta k+\delta \int_{0}^{+\infty}\left(\beta+\gamma_{2}(\theta) e^{-b \theta} \Gamma(\theta) d \theta\right)\right] \limsup _{t \rightarrow \infty} u(t) e^{-(b+\delta+\alpha) \tau} d \tau \\
= & {\left[\beta k+\delta \int_{0}^{+\infty}\left(\beta+\gamma_{2}(\theta) e^{-b \theta} \Gamma(\theta) d \theta\right)\right] \int_{0}^{+\infty} e^{-(b+\delta+\alpha) \tau} d \tau \limsup u(t) } \\
\leq & \frac{\left.k \beta+\delta \int_{0}^{\infty}\left(\beta+\gamma_{2}(\theta)\right) e^{-b \theta} \Gamma(\theta) d \theta\right)}{b+\delta+\alpha} \limsup u(t) \\
= & R_{0} \limsup _{t \rightarrow \infty} u(t) .
\end{aligned}
$$

Since $R_{0}<1$, the above inequality can only hold if $\limsup _{t \rightarrow \infty} u(t)=0$. From (3.18), we also have $\lim \sup _{t \rightarrow \infty} v(\theta, t)=0$ for all $\theta$ fixed. This completes the proof.

\subsection{The Locally Asymptotical Stability of the Endemic Equilibrium}

In this subsection, we will show that the endemic equilibrium is locally asymptotically stable as long as it exists.

Theorem 3.3. If $R_{0}>1$, the endemic equilibrium $M^{*}$ is locally asymptotically stable (LAS). 
Proof. Set

$$
s=s^{*}+\bar{x}, \quad u=u^{*}+\bar{y}, \quad v=v^{*}+\bar{z} .
$$

Plug it into the system (3.4) and ignore the high-degree terms, then we get the linearization around $M^{*}$ :

$$
\begin{gathered}
\bar{x}^{\prime}=-b \bar{x}-\lambda^{*} \bar{x}-\bar{\lambda} s^{*}+\alpha \bar{y}+\int_{0}^{\infty} r_{1}(\theta) \bar{z}(\theta, t) d \theta, \\
\bar{y}^{\prime}=\lambda^{*} \bar{x}+\bar{\lambda} s^{*}-(b+\delta+\alpha) \bar{y}+\int_{0}^{\infty} \gamma_{2}(\theta) \bar{z}(\theta, t) d \theta, \\
\bar{z}_{\theta}+\bar{z}_{t}=-\left(b+\gamma_{1}(\theta)+\gamma_{2}(\theta)\right) \bar{z}(\theta, t), \\
\bar{z}(0, t)=\delta \bar{y} \\
\lambda^{*}=\beta\left(k u^{*}+\int_{0}^{\infty} v *(\theta) d \theta\right), \\
\bar{\lambda}=\beta\left(k \bar{y}+\int_{0}^{\infty} \bar{z}(\theta, t) d \theta\right) .
\end{gathered}
$$

Looking for exponential solutions in (3.24), that is, solutions of the form

$$
\bar{x}=e^{\omega t} x, \quad \bar{y}=e^{\omega t} y, \quad \bar{z}=e^{\omega t} z(\theta),
$$

where $\omega$ is a constant. Substituting it into (3.24), we have

$$
\begin{gathered}
\omega x=-b x-\lambda^{*} x-\tilde{\lambda} s^{*}+\alpha y+\int_{0}^{\infty} \gamma_{1}(\theta) z(\theta) d \theta \\
\omega y=\lambda^{*} x+\tilde{\lambda} s^{*}-(b+\delta+\alpha) y+\int_{0}^{\infty} \gamma_{2}(\theta) z(\theta) d \theta \\
\omega z+z_{\theta}=-\left(b+\gamma_{1}(\theta)+\gamma_{2}(\theta)\right) z(\theta) \\
z(0)=\delta y \\
\lambda^{*}=\beta\left(k u^{*}+\int_{0}^{\infty} v^{*}(\theta) d \theta\right) \\
\tilde{\lambda}=\beta\left(k y+\int_{0}^{\infty} z(\theta) d \theta\right)
\end{gathered}
$$

where

$$
x+y+\int_{0}^{\infty} z(\theta) d \theta=0 .
$$


From the third and the fourth equations in (3.26), we get $z(\theta)=\delta y e^{-(\omega+b) \theta} \Gamma(\theta)$. Hence (3.26) can be rewritten as:

$$
\begin{gathered}
\omega x=-b x-\lambda^{*} x-\tilde{\lambda} s^{*}+\alpha y+\delta y \int_{0}^{\infty} \gamma_{1}(\theta) e^{-(\omega+b) \theta} \Gamma(\theta) d \theta, \\
\omega y=\lambda^{*} x+\tilde{\lambda} s^{*}-(b+\delta+\alpha) y+\delta y \int_{0}^{\infty} \gamma_{2}(\theta) e^{-(\omega+b) \theta} \Gamma(\theta) d \theta,
\end{gathered}
$$

and the condition (3.27) becomes

$$
x+y+\delta y \int_{0}^{\infty} e^{-(\omega+b) \theta} \Gamma(\theta) d \theta=0 .
$$

In fact, one of the three equations of (3.28) and (3.29) is a consequence of the other two. In particular, adding the equations in (3.28) and using the fact that

$$
\int_{0}^{\infty}\left(\omega+b+\gamma_{1}(\theta)+\gamma_{2}(\theta)\right) e^{-(\omega+b) \theta} \Gamma(\theta) d \theta=1
$$

we have (3.29). equation:

From the second equation in (3.28) and (3.29) and cancelling $y$ we get the characteristic

$$
\omega+b+\delta+\alpha+\lambda^{*}\left(1+\delta \int_{0}^{\infty} e^{-(\omega+b) \theta} \Gamma(\theta) d \theta\right)=s^{*} k \beta+\int_{0}^{\infty}\left[s^{*} \beta \delta+\delta \gamma_{2}(\theta)\right] e^{-(\omega+b) \theta} \Gamma(\theta) d \theta
$$

Assume that $\omega=\xi+\eta i$, and the equation for the real part $\xi$ becomes

$$
\begin{aligned}
\xi+b+ & \delta+\alpha+\lambda^{*}\left(1+\delta \int_{0}^{\infty} e^{-(\xi+b) \theta} \Gamma(\theta) \cos (\eta \theta) d \theta\right) \\
& =s^{*} k \beta+\int_{0}^{\infty}\left[s^{*} \beta \delta+\delta \gamma_{2}(\theta)\right] e^{-(\xi+b) \theta} \Gamma(\theta) \cos (\eta \theta) d \theta .
\end{aligned}
$$

If $\xi \geq 0$, then the left hand side of (3.32) is strictly larger than $b+\delta+\alpha$, and from (3.8) we note that the right hand side of (3.32) is just $b+\delta+\alpha$. This is a contradiction, so $\xi<0$, that is, any complex eigenvalue has negative real part, then we can say the endemic equilibrium $M^{*}$ is locally asymptotically stable. The proof is complete.

\section{Simulations}

In this section, the system (2.9) is simulated for various sets of parameters and we will find that the results are consistent with the analytical results. In Figure 2, we give an example showing that the disease-free equilibrium $Q_{0}$ is stable when $R_{0}<1$. Figure 3 illustrate that 
Estimated TB incidence, prevalence and mortality, 2008.

\begin{tabular}{|c|c|c|c|c|c|c|c|}
\hline \multirow[b]{2}{*}{ WHO region } & \multicolumn{3}{|c|}{ Incidence $^{1}$} & \multicolumn{2}{|l|}{ Prevalence $^{2}$} & \multicolumn{2}{|l|}{ Mortality $^{3}$} \\
\hline & $\begin{array}{l}\text { no. in } \\
\text { thousands }\end{array}$ & $\begin{array}{c}\% \text { of global } \\
\text { total }\end{array}$ & $\begin{array}{c}\text { Rate per } \\
100000 \\
\text { pop }^{3}\end{array}$ & $\begin{array}{l}\text { no. in } \\
\text { thousands }\end{array}$ & $\begin{array}{c}\text { Rate per } \\
100000 \\
\text { pop }\end{array}$ & $\begin{array}{l}\text { no. in } \\
\text { thousands }\end{array}$ & $\begin{array}{c}\text { Rate per } \\
100000 \\
\text { pop }\end{array}$ \\
\hline Africa & 2828 & $30 \%$ & 351 & 3809 & 473 & 385 & 48 \\
\hline The Americas & 282 & $3 \%$ & 31 & 221 & 24 & 29 & 3 \\
\hline $\begin{array}{l}\text { Eastern } \\
\text { Mediterranean }\end{array}$ & 675 & $7 \%$ & 115 & 929 & 159 & 115 & 20 \\
\hline Europe & 425 & $5 \%$ & 48 & 322 & 36 & 55 & 6 \\
\hline $\begin{array}{l}\text { South-East } \\
\text { Asia }\end{array}$ & 3213 & $34 \%$ & 183 & 3805 & 216 & 477 & 27 \\
\hline $\begin{array}{l}\text { Western } \\
\text { Pacific }\end{array}$ & 1946 & $21 \%$ & 109 & 2007 & 112 & 261 & 15 \\
\hline Global total & 9369 & $100 \%$ & 139 & 11093 & 164 & 1322 & 20 \\
\hline
\end{tabular}

(a)

Rate per 100000 population

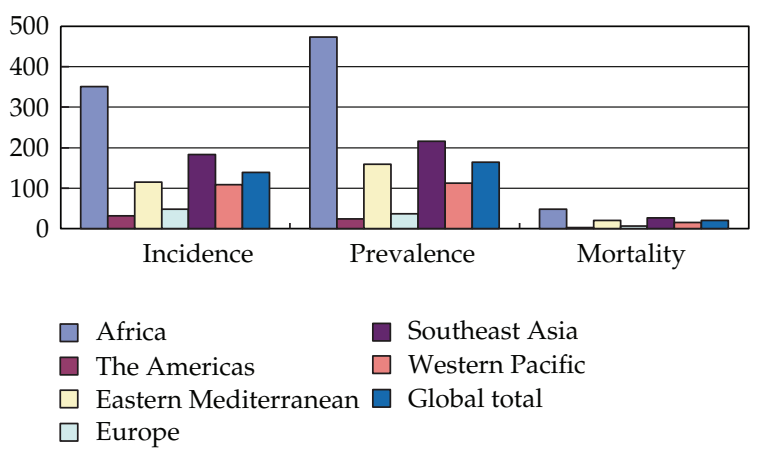

(b)

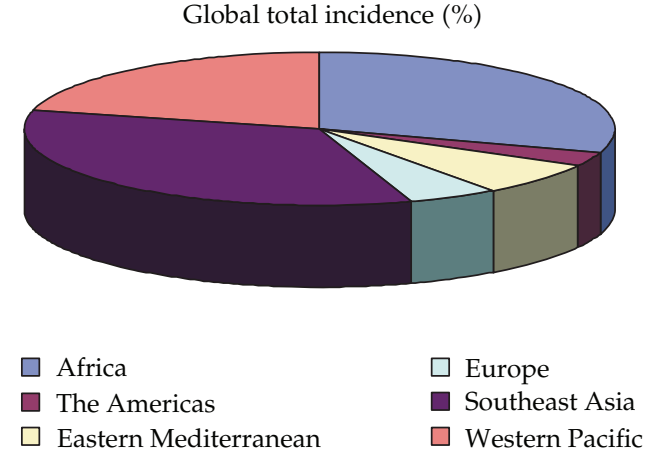

(c)

Figure 1: The estimated TB incidence, prevalence, and mortality in differen regions in 2008.

there exists an endemic equilibrium $Q^{*}$, which is stable when $R_{0}>1$. The difference between two groups of parameter values only lie in $k$ : the coefficient of reduction of infection. Figure 1 represents when we omit infectivity in latent period, and Figure 2 gives a very different result at the same case if we consider weaker infectivity in latent period, which means that it would make a big difference to the prevalence of TB whether considering infectivity in latent period or not. To find better control strategies for TB infection, we would like to see what parameters can reduce the basic reproduction number $R_{0}$. In Figure 4(a) we can see that $R_{0}$ decreases if $\gamma_{1}$ increases or $\alpha$ increases. From Figure $4(\mathrm{~b})$ we can find that $R_{0}$ decreases if $b$ increases, or $\beta$ decreases. Although they all act to decrease the basic reproduction numbers, which can be seen by analysis above, it often does so by different amounts. When it comes to complicated relations between $R_{0}$ and $\delta$ or $\gamma_{2}$, we can see from Figure 5(a) that the basic reproduction number $R_{0}$ is a increasing function of $\delta$ and decreasing function of $\gamma_{2}$, for $(b+\alpha)\left(\beta+\gamma_{2}\right)-(b+$ $\left.\gamma_{1}+\gamma_{2}\right) k \beta>0$ and $b+\gamma_{1}-\beta<0$, which coincides with the analysis above. In Figure 5(b) we assume $k=0.04$, which implies the sign of $(b+\alpha)\left(\beta+\gamma_{2}\right)-\left(b+\gamma_{1}+\gamma_{2}\right) k \beta$ is alterable, thus in 


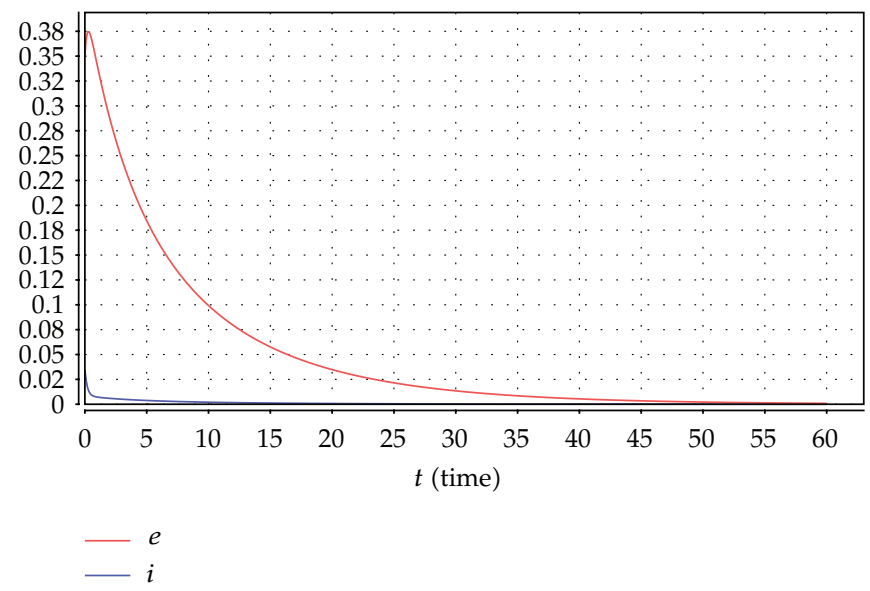

Figure 2: Phase plot of $e$ versus $i$ showing the disease-free equilibrium $Q_{0}$ which is stable when $R_{0}<1$ for the parameter values $\alpha=0.3, b=0.0143, \beta=13, \delta=0.1, \gamma_{1}=2, \gamma_{2}=3, k=0, e_{0}=0.35, i_{0}=0.035$. Based on these parameters, $R_{0} \approx 0.77<1$.

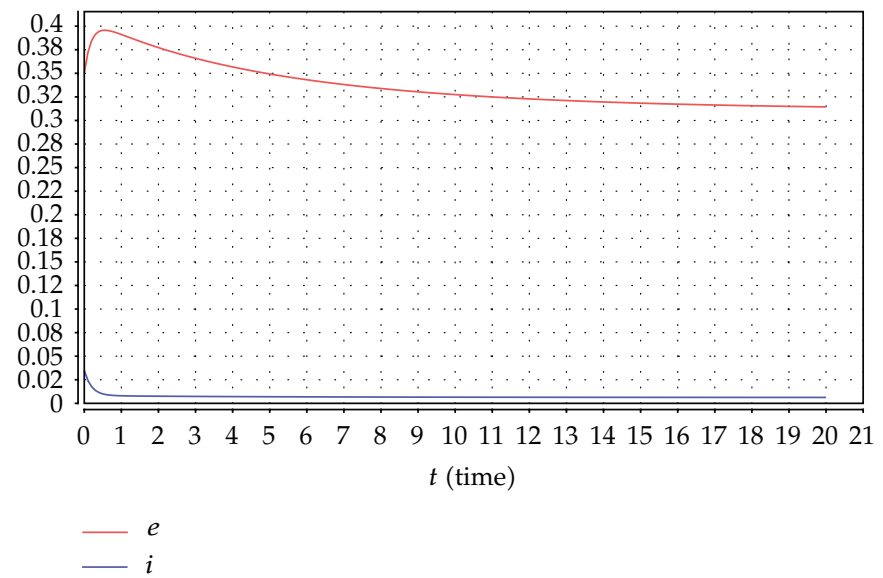

Figure 3: Phase plot of $e$ versus $i$ showing the existence of endemic equilibrium $Q^{*}$ which is stable when $R_{0}>1$, for the parameter values for the parameter values $k=0.02$ and the others are the same as Figure 2 . Based on these parameters, $R_{0} \approx 1.398>1$.

some region $R_{0}$ is a decreasing function of $\delta$, but others are increasing. So to find better control strategies for TB infection, we should consider all factors and its weight comprehensively.

\section{Discussion}

In this paper we formulate a new model of a common disease, that is, TB. We assume that people in latent period has weaker infectivity, and those both in latent period and infective period can receive successful or unsuccessful treatment. We firstly introduce a simple ODEs model, and prove that sustained oscillations are not possible because the endemic proportions either approach the disease-free equilibrium or an endemic equilibrium. For the behavior of the proportions does not give us much insight on the behavior of the total 


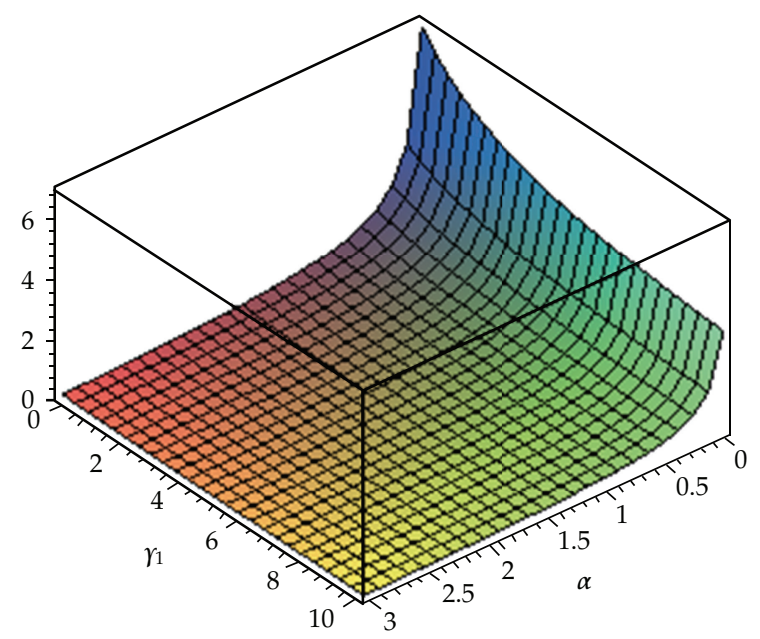

(a)

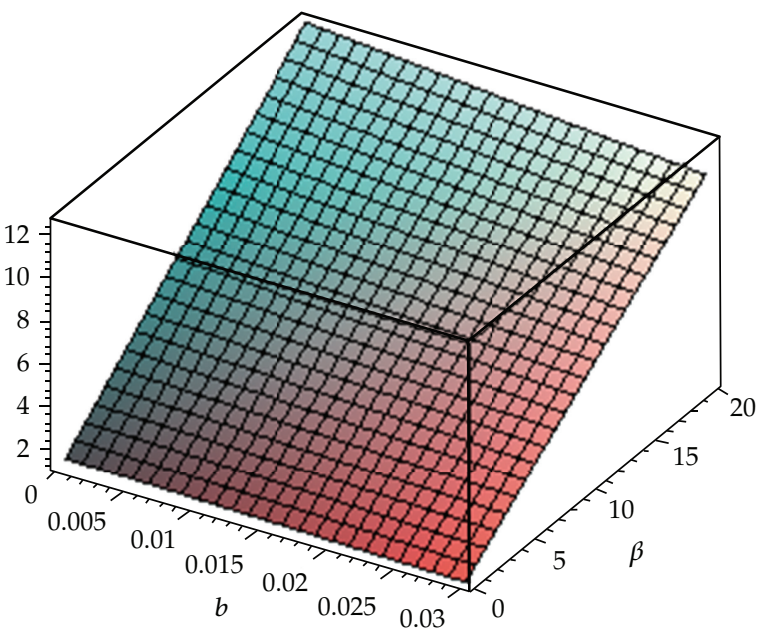

(b)

Figure 4: The graphs of the basic reproduction number $R_{0}$ in terms of some parameters: (a) $R_{0}$ in terms of $\alpha$ and $\gamma_{1}$, for the remaining parameter values are the same as Figure 3; (b) $R_{0}$ as a function of $b$ and $\beta$, for the remaining parameter values are the same as Figure 3.

number, we also define a basic replacement ratio. Then we consider an extension of the ODEs model from the previous section in which the infective stage is stratified by the age-sinceinfection, that is, the time spent in the infective stage. In this section we show that the diseasefree equilibrium is globally asymptotically stable if $R_{0}<1$ and the endemic equilibrium is locally asymptotically stable if $R_{0}<1$. However, it is regretful that in this paper we omit the disease-induced mortality and do not discuss persistence of the disease in PDE model. The proof of uniformly strong persistence involves complicated theory, and the reader interested in it can refer to $[25,26]$. 


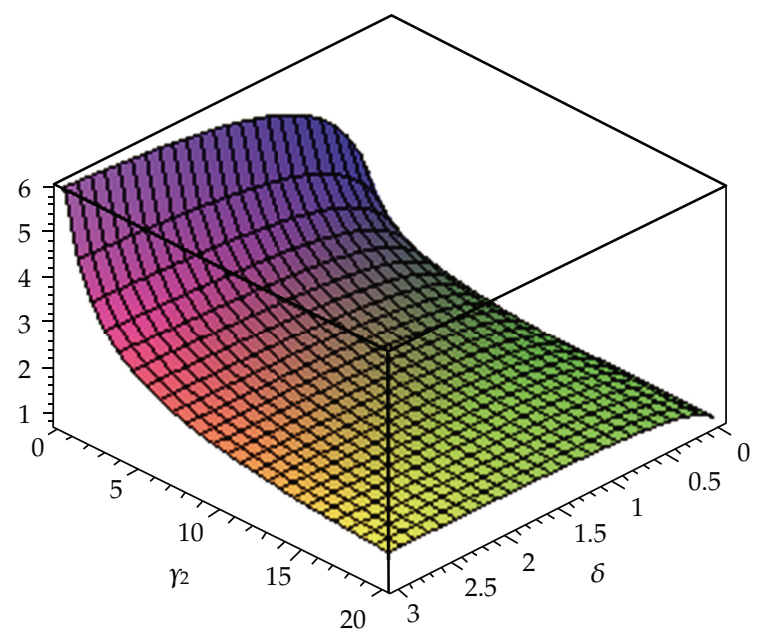

(a)

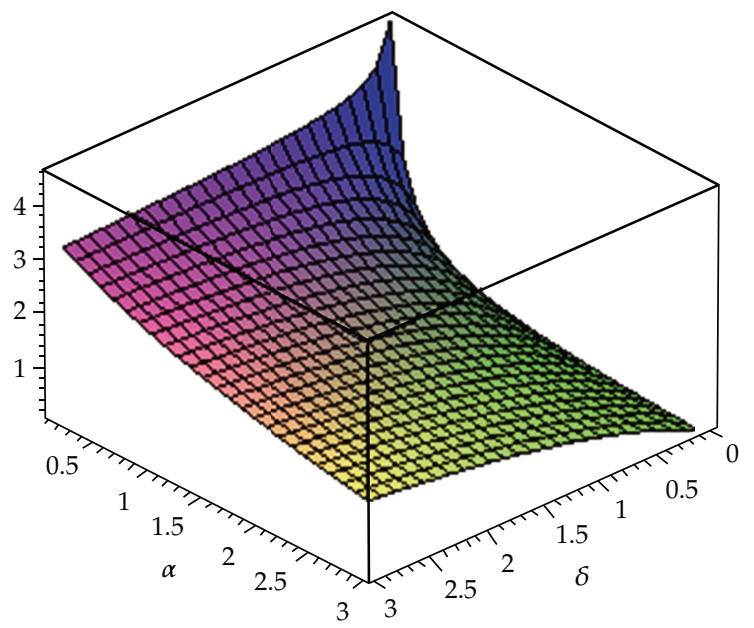

(b)

Figure 5: The graphs of the basic reproduction number $R_{0}$ in terms of some parameters: (c) $R_{0}$ in terms of $\beta$ and $\delta$, for the remaining parameter values are the same as Figure 3; (d) $R_{0}$ in terms of $\alpha$ and $\delta$, for the remaining parameter values are $k=0.04$, and the others are the same as Figure 2 .

\section{Acknowledgments}

The authors greatly appreciate the anonymous referee and the handing editor Professor Zhen Jin for their valuable comments which help to improve the paper. This paper is supported by the NSF of China (no. 10971178) and NSF of Henan Province (no. 112300410238, no. 112300410058).

\section{References}

[1] B. Song, C. Castillo-Chavez, and J. P. Aparicio, "Tuberculosis models with fast and slow dynamics: the role of close and casual contacts," Mathematical Biosciences, vol. 180, pp. 187-205, 2002. 
[2] C. Dye, K. Floyd, and M. Uplekar, "World health report: global tuber-culosis control: surveillance, planning, financing," WHO/HTM/TB/ 2008.393, World Health organization, 2008.

[3] C. Castillo-Chavez and Z. Feng, "To treat or not to treat: the case of tuberculosis," Journal of Mathematical Biology, vol. 35, no. 6, pp. 629-656, 1997.

[4] S. M. Blower, A. R. McLean, T. C. Porco et al., "The intrinsic transmission dynamics of tuberculosis epidemics," Nature Medicine, vol. 1, no. 8, pp. 815-821, 1995.

[5] C. P. Bhunu, W. Garira, and Z. Mukandavire, "Modeling HIV/AIDS and tuberculosis coinfection," Bulletin of Mathematical Biology, vol. 71, no. 7, pp. 1745-1780, 2009.

[6] S. M. Blower and T. Chou, "Modeling the emergence of the "hot zones": tuberculosis and the amplification dynamics of drug resistance," Nature Medicine, vol. 10, no. 10, pp. 1111-1116, 2004.

[7] T. Cohen and M. Murray, "Modeling epidemics of multidrug-resistant tuberculosis of heterogeneous fitness," Nature Medicine, vol. 10, no. 10, pp. 1117-1121, 2004.

[8] P. Rodrigues, M. G.M. Gomes, and C. Rebelo, "Drug resistance in tuberculosis-a reinfection model," Theoretical Population Biology, vol. 71, no. 2, pp. 196-212, 2007.

[9] E. Vynnycky and P. E.M. Fine, "The natural history of tuberculosis: the implications of age-dependent risks of disease and the role of reinfection," Epidemiology and Infection, vol. 119, no. 2, pp. 183-201, 1997.

[10] C. P. Bhunu, W. Garira, Z. Mukandavire, and M. Zimba, "Tuberculosis transmission model with chemoprophylaxis and treatment," Bulletin of Mathematical Biology, vol. 70, no. 4, pp. 1163-1191, 2008.

[11] O. Sharomi, C. N. Podder, A. B. Gumel, and B. Song, "Mathematical analysis of the transmission dynamics of HIV/TB coinfection in the presence of treatment," Mathematical Biosciences and Engineering, vol. 5, no. 1, pp. 145-174, 2008.

[12] L.-I.W. Roeger, Z. Feng, and C. Castillo-Chavez, "Modeling TB and HIV co-infections," Mathematical Biosciences and Engineering, vol. 6, no. 4, pp. 815-837, 2009.

[13] N. Bacaër, R. Ouifki, C. Pretorius, R. Wood, and B. Williams, “Modeling the joint epidemics of TB and HIV in a South African township," Journal of Mathematical Biology, vol. 57, no. 4, pp. 557-593, 2008.

[14] Y. Zhou, K. Khan, Z. Feng, and J. Wu, "Projection of tuberculosis incidence with increasing immigration trends," Journal of Theoretical Biology, vol. 254, no. 2, pp. 215-228, 2008.

[15] S. Akhtar and H. G. H. H. Mohammad, "Seasonality in pulmonary tuberculosis among migrant workers entering Kuwait," BMC Infectious Diseases, vol. 8, 2008.

[16] T. C. Porco and S. M. Blower, "Quantifying the intrinsic transmission dynamics of tuberculosis," Theoretical Population Biology, vol. 54, no. 2, pp. 117-132, 1998.

[17] D. E. Snider, M. Raviglione, and A. Kochi, "Global burden of tuberculosis," in Tuberculosis Pathogenisis and Control, B. Bloom, Ed., ASM, Washington, DC, USA, 1994.

[18] A. S. Douglas, D. P. Strachan, and J. D. Maxwell, "Seasonality of tuberculosis: the reverse of other respiratory diseases in the UK," Thorax, vol. 51, no. 9, pp. 944-946, 1996.

[19] L. Liu, X.-Q. Zhao, and Y. Zhou, "A tuberculosis model with seasonality," Bulletin of Mathematical Biology, vol. 72, no. 4, pp. 931-952, 2010.

[20] B. Fang and X. Z. Li, "Stability of an age-structured MSEIS epidemic model with infectivity in latent period," Acta Mathematicae Applicatae Sinica, vol. 31, no. 1, pp. 110-125, 2008.

[21] L. Guihua and J. Zhen, "Global stability of an SEI epidemic model," Chaos, Solitons and Fractals, vol. 21, no. 4, pp. 925-931, 2004.

[22] G. Li and J. Zhen, "Global stability of an SEI epidemic model with general contact rate," Chaos, Solitons and Fractals, vol. 23, no. 3, pp. 997-1004, 2005.

[23] G. Li and Z. Jin, "Global stability of a SEIR epidemic model with infectious force in latent, infected and immune period," Chaos, Solitons and Fractals, vol. 25, no. 5, pp. 1177-1184, 2005.

[24] T. J. Case, An Illustrated Guide to Theoretical Ecology, Oxford University, New York, NY, USA, 2000.

[25] H. R. Thieme, "Uniform weak implies uniform strong persistence for non-autonomous semiflows," Proceedings of the American Mathematical Society, vol. 127, no. 8, pp. 2395-2403, 1999.

[26] H. R. Thieme, Y. Takeuchi, Y. Iwasa, and K. Sato, "Pathogen competition and coexistence and the evolution of virulence," in Mathematics for Life Sciences and Medicine, vol. 123, 2007. 


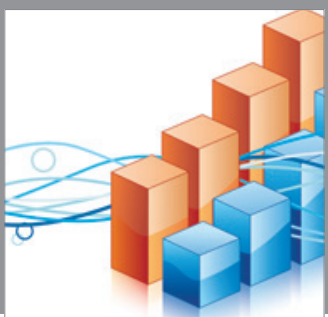

Advances in

Operations Research

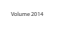

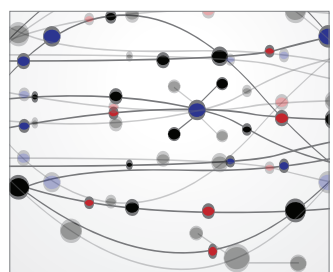

\section{The Scientific} World Journal
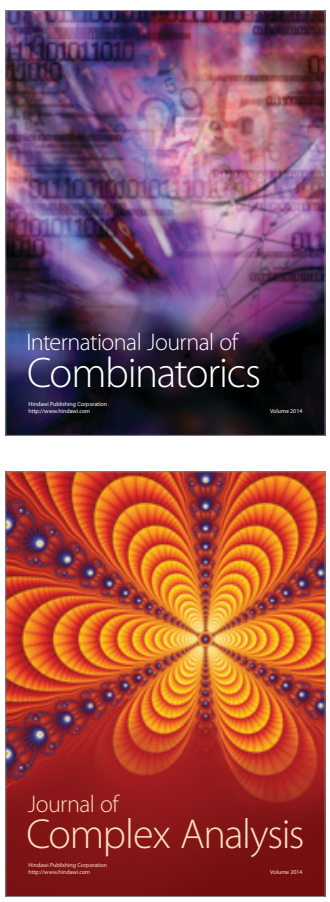

International Journal of

Mathematics and

Mathematical

Sciences
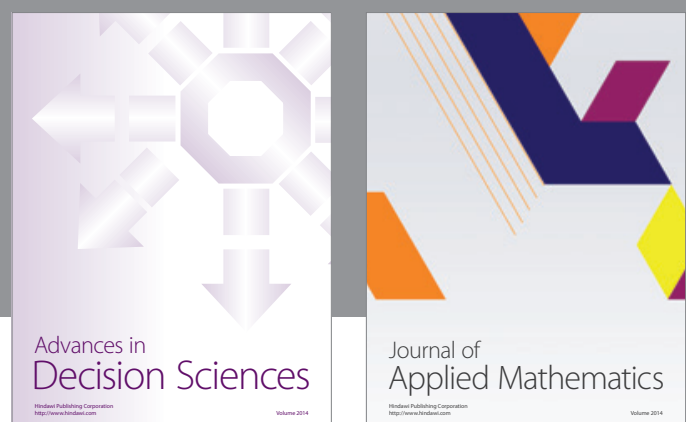

Journal of

Applied Mathematics
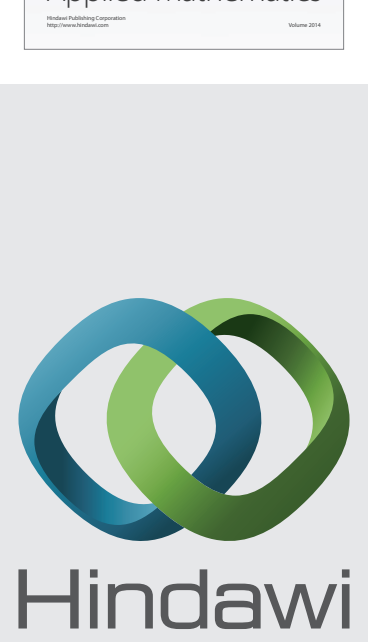

Submit your manuscripts at http://www.hindawi.com
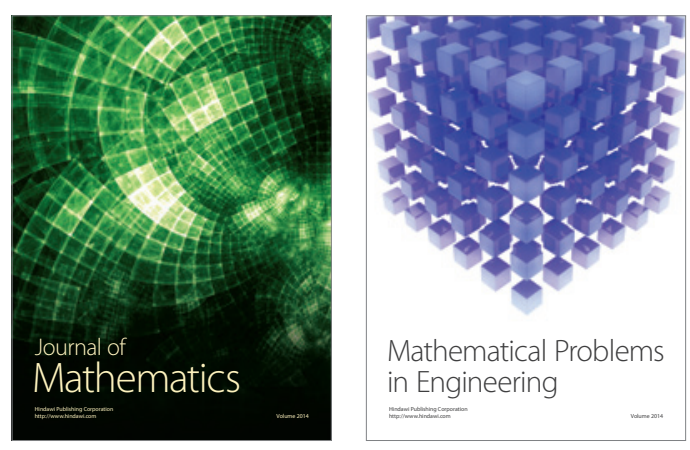

Mathematical Problems in Engineering
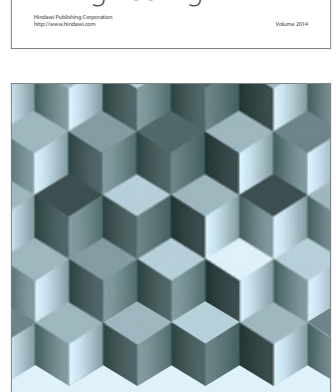

Journal of

Function Spaces
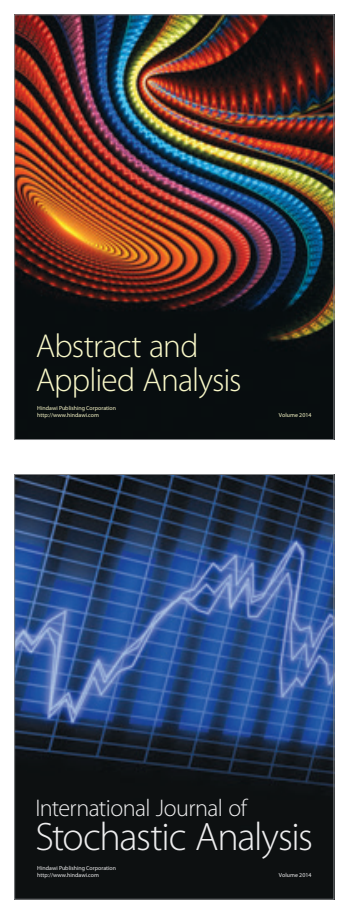

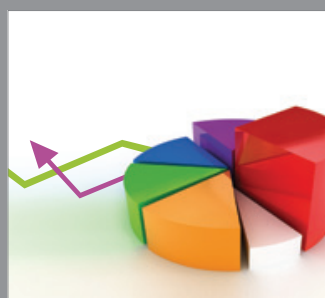

ournal of

Probability and Statistics

Promensencen
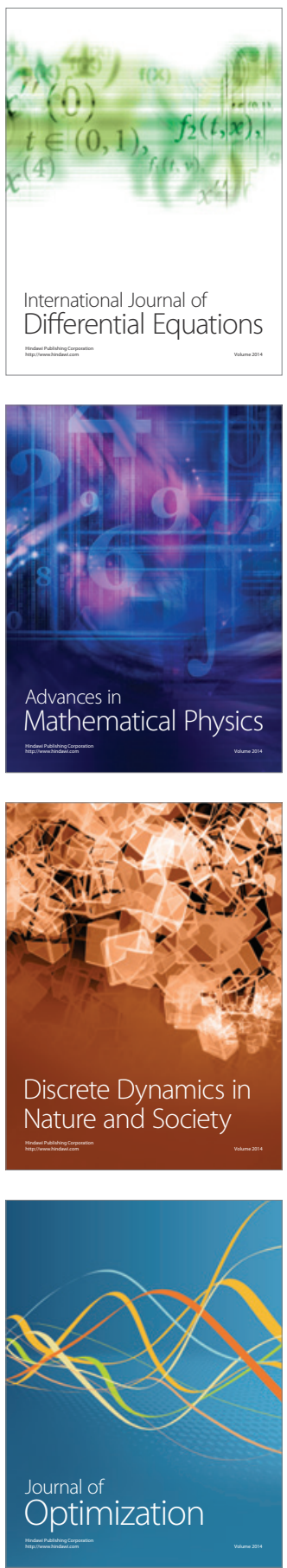